\title{
GPR application in Civil Engineering to search and detect underground Networks
}

\author{
Tahar Bachiri $^{1, a}$, Gamil Alsharahi ${ }^{2}$, Abdellatif Khamlichi ${ }^{3}$, Mohammed Bezzazi $^{4}$, Ahmed Faize $^{5}$ \\ ${ }^{1}$ Department of Mechanical and Civil Engineering, Faculty of Sciences and Technology, Tangier 91001, \\ University Abdelmalek Essaadi, Morocco, tbachiri@uae.ac.ma ( ${ }^{\text {a Corresponding Auteur) }}$ \\ ${ }^{2}$ Department of Physics, Faculty Polydisiplinarly Nador, Mohammed First University, Morocco. \\ alsharahigamil@gmail.com \\ ${ }^{3}$ Department STIC, National School of Applied Sciences, University Abdelmalek Essaadi, Tetouan 93030, \\ Morocco, khamlichi7@yahoo.es \\ ${ }^{4}$ Department of Mechanical and Civil Engineering, Faculty of Sciences and Technology, Tangier 91001, \\ University Abdelmalek Essaadi, Morocco, bezzazi@hotmail.com \\ ${ }^{5}$ Department of Physics, Faculty Polydisiplinarly Nador, Mohammed First University, Morocco. \\ ahmedfaize6@hotmail.com
}

\begin{abstract}
The industrial development and the intensive use of urban space lead to several problems in the management and operation of public services such as drinking water networks, sewer, telephone, gas, electricity, street lighting, and various cables and pipes that are buried either at footpaths or roadways. Damage or malfunction of pre-existing public services can potentially cause danger to humans. In order to minimize risks, GPR profiling was constructed as a tool of advanced non-destructive technologies leading to practical solutions to detect buried networks and reduce problems related to the destruction of existing underground utilities. The main objective of this study is to search and identify the various hidden systems to map them and indicate them on-site to avoid possible incidents. The purpose of this operation is to produce GPR profiles on the surface to be examined, while respecting a fairly regular spacing between patterns, using non-destructive techniques such as the GPR and the radio detector.
\end{abstract}

Key words: Buried networks, GPR profiling, non-destructive technologies, radio detector.

\section{INTRODUCTION}

The network system of pre-existing public services, which includes underground hoppers and tunnels, drinking water and sewer pipes, gas pipes, storm water pipes, telecommunications cables, electricity and street lighting cables is a diverse, complex and often poorly defined network. Due to a need for knowledge of underground utility development, civil construction projects can easily cause, for example, gas explosion, road collapse, water leaks, infiltration of the road surface, cavities, fragile areas and void spaces [1], [2], [3], [4], [5], [6].
The GPR is an instrument that is used for subsurface mapping using high-frequency Electromagnetic (EM) waves. An attempt has been made to use GPR to detect and locate buried objects. Via using GPR images, we can identify metallic and non-metallic buried utilities like primary mines, pipes, cables, etc. Furthermore, the GPR images can be used to obtain more quantitative information about the location, shape and size of buried objects reflecting the GPR signals [7], [8]. In practice, the operator in charge of the geo-detection of underground networks proceeds in several steps. The first step is to collect the maximum of information about the ground and the environment together with its knowledge about the standards of installation of underground networks and the use of detection methods.

The GPR method has advantages such as its speed of application, safety, convenience, low cost, no trench, anti-interference [9]. This method was used to map utilities in the basement to guide subway construction [10], to locate buried pipes and to monitor leaks [110], [12], to estimate soil moisture content [13], to assess road test sites and to test defects in technical structures [14], [15]. Based on the analysis of several radargrams, a geometric relationship was determined to estimate the diameter and depth of the buried pipe [16]. The GPR has also found wider applicability as an electromagnetic device for assessing subsurface characteristics of transportation infrastructure [17]. Given the practical utility of these parameters in the field of civil engineering, it has been demonstrated through numerical analysis that in order to operate the detection by the GPR, it is necessary to choose correctly the central frequency of the antennas [18], [19], [20].

There is a wide range of assessments of the condition and dangers of underground utilities such as voids, water infiltration, leakage, groundwater flow, electrical cables and gas explosions that often cause ground collapse and, in some cases, landslides. The cause is a physicochemical process 
Tahar Bachiri et al., International Journal of Emerging Trends in Engineering Research, 8(5), May 2020, 1839- 1844

triggered by material degradation and damage during excavation. Therefore, the diagnosis of voids, leaks of drinking water or sewers and the assessment of the extent of infiltration are necessary to minimize damage. Several reasons can be given to justify mapping water infiltration, leakage and vacuum in a city [21]:

- Water is the most influential factor in slowing the speed of motion of GPR waves, causing attenuation in dielectric building materials, and absorbing the high-frequency component of the wave due to the dielectric polarization mechanism [22];

- The GPR wave moves in the material without physical contact of the sensors with the pipes, such as valves, as required by other acoustic methods such as the noise recorder and leakage correlation;

- Different depths of water pipe buried in the road or slope can be reached by adopting the GPR antenna in different central frequencies. For example, gradients at the scale of tens of meters can be studied by an antenna with central frequencies ranging from 100 to $500 \mathrm{Mhz}$, and then dikes and meter-scale roads are in the frequency range of 400 to $900 \mathrm{Mhz}$ [23].

One of the most complicated civil engineering applications is the positioning and mapping of urban utilities by the GPR. This is because GPR is often used to position and map underground elements such as pipes, cables, barrels, reservoirs and graves [24], [25]. In addition, the difficulties that can result from a single 2D B-scan, any hyperbolic reflection can be either a public service or other anomalies with dielectric contrast like rocks. On the contrary, according to some authors, efficient and large-scale data collection and 3D mapping are particularly important for the utility survey [26], [27]. Underground pipeline exploration has become an essential aspect of underground urban engineering construction projects [1].

To date, many efforts and literature have focused on the hazards that can occur in the development of underground utility systems. As part of this study, we conducted an in-depth study of the location of buried sewer lines and the detection of anomalies using a GPR. The inspection was performed on actual public equipment. The GPR method was combined with the radio detector method. In this document, the details of the study methodology, GPR instrumentation, image analysis, the drawing of the proofing diagrams and the results of the GPR study will be presented and discussed.

\section{MATERIALS AND METHODS}

In order to carry out our mission, we implemented the following means:

- GPR equipped with a $400 \mathrm{Mhz}$ antenna with a depth of investigation of about $3 \mathrm{~m}$;

- Radio detector.

\subsection{GPR method}

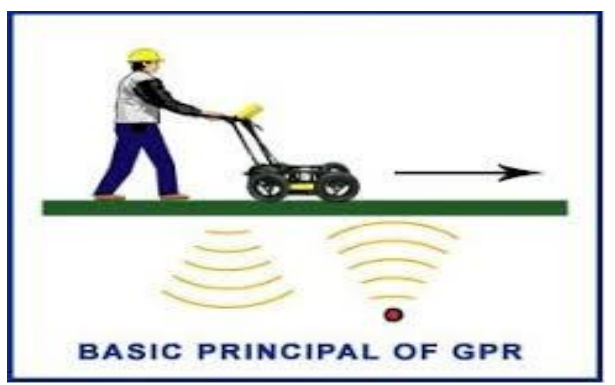

Figure 1: GPR hardware used

The Ground Penetrating Radar (GPR) works on the principle of studying the propagation of an electromagnetic wave in the studied environment. The system sends a very short wave (pulse) into the material and records the amplitude and time of arrival of each reflected wave (see Figure 1). The reflections are produced in the right of any change in the conduction properties of the electrical current of the medium (dielectric constant). The amplitude of the reflection is determined by the dielectric permittivity contrast between the encasing and the target.

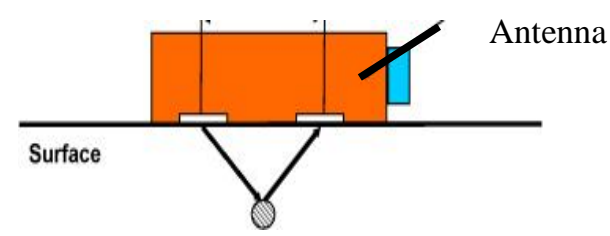

Figure 2: principle of GPR inspection

Some of the energy sent also continues to propagate into the medium until it is too attenuated to be detected. The attenuation of the signal is thus highly variable and depends largely on the electrical conductivity of the materials. Terrain with high electrical conductivity will attenuate GPR waves very strongly and vice versa.

The metal is considered a total reflector and is therefore easily detectable. In addition, objects located directly under a metal object cannot be detected. The GPR wave is not emitted in a straight line from the antenna but describes an emission cone of a known width (see Figure 2). The time of the wave path to the edge of this cone is greater than that at the center of the antenna; this is the origin of the form of hyperbole characteristic of a point object. The target is located at the top of this signature (see Figure 3).

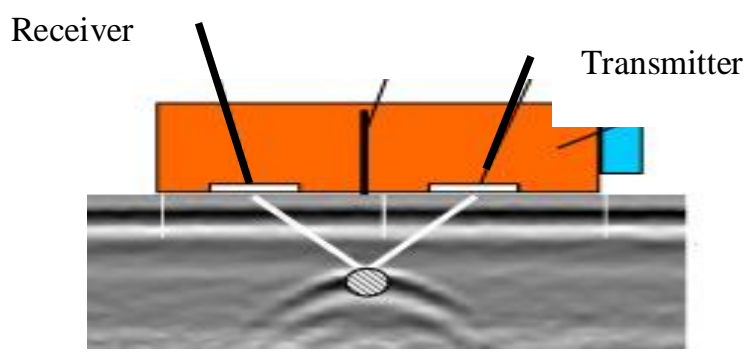

Figure 3: the top of the hyperbole marks the position of the target 
Tahar Bachiri et al., International Journal of Emerging Trends in Engineering Research, 8(5), May 2020, 1839- 1844

The most important parameter that controls the depth of electromagnetic waves is the transmitter antenna frequency. Indeed, the choice of the operating frequency of the GPR antenna must be carefully made to ensure a favorable compromise between detection depth and spatial resolution. For vertical resolution, which allows for the proper geometry of buried objects, it depends on the central frequency of the antenna and the GPR wave speed.

The horizontal resolution, which depends mainly on the number of traces and the depth of the reflector, indicates the minimum distance between two horizontal reflectors to be detected separately. The horizontal $\left(\mathrm{R}_{\mathrm{H}}\right)$ and vertical $\left(\mathrm{R}_{\mathrm{V}}\right)$ resolutions of each antenna are as follows [11], [28], [29] :

$$
\mathrm{R}_{\mathrm{H}}=2\left(\frac{\lambda^{2}}{4}+\mathrm{d} \cdot \lambda\right)^{0,5}
$$

Where, $\lambda$ is the wavelength and $d$ is the vertical distance between the antenna and the reflector surface.

$$
\mathrm{R}_{\mathrm{V}}=\frac{1,39 \cdot v \cdot \Delta \mathrm{t}}{2}
$$

Where, $v$ is the GPR wave speed $(\mathrm{cm} / \mathrm{ns})$ and $\Delta \mathrm{t}$ is the effective duration of the GPR pulse (ns).

To define the total time window or bidirectional travel time $\left(\mathrm{T}_{\mathrm{W}}\right)$, it is necessary to consider the optimal number of samples $(\mathrm{N})$ as follows [11], [30]:

$$
\mathrm{T}_{\mathrm{W}}=1,3 \cdot \frac{2 \cdot \mathrm{d}}{v}
$$

Where, $\mathrm{d}$ is the vertical distance between the antenna and the reflector surface and $\mathrm{v}$ is the GPR wave speed.

The minimum number of samples (Nmin) is determined by the following relation(4) [11], [30]:

$$
\mathrm{N}_{\min }=\frac{6 \cdot f \cdot \mathrm{T}_{\mathrm{W}}}{1000}
$$

Where $f$ is the central frequency of the antenna in Mhz and that is the bidirectional travel time in ns.

Spacing or trace intervals are expressed according to this relation (5) [11], [30]:

$$
\Delta \mathrm{x}=\frac{\mathrm{c}}{4 \cdot f \cdot \sqrt{\varepsilon}}=\frac{75}{f \cdot \sqrt{\varepsilon}}(\mathrm{m})
$$

Where $\varepsilon$ is the dielectric constant of the host environment. Based on the principle of transmission and reflection of electromagnetic waves, the equation of the propagation distance of the electromagnetic wave is equation (6) [31] :

$$
\mathrm{L}=\mathrm{v} \cdot \mathrm{t}=\left(\frac{\mathrm{c}}{\sqrt{\varepsilon}}\right) \cdot \mathrm{t}
$$

Where $\mathrm{L}$ is the propagation distance of the electromagnetic wave; $\mathrm{v}$ is the speed of the electromagnetic wave in a certain medium, $\mathrm{c}$ is the speed of the electromagnetic wave, $\varepsilon$ is the relative dielectric constant of the transmission medium, $t$ is the transmission time.

\subsection{Material used}

The material used is branded VIVAX VLOC Pro2. This material is based on the transmission and reception of electromagnetic waves with a frequency between 50 and 33000 Hertz. It can be implemented passively (mainly for the detection of $50 \mathrm{~Hz}$ electrical cables) or activities for the detection of metal networks. For active detection, the transmission of the electromagnetic signal to the network is done by connecting a clamp-meter if the network is accessible or by induction if one does not have access to the network.

In order to simplify the presentation of the data, an area of [1500x1000] cm was selected to perform on-site GPR profiles to cover the entire area to be examined. The GPR equipment is equipped with a $400 \mathrm{Mhz}$ antenna, which allows the deep search up to $3 \mathrm{~m}$. the ground dielectric constant of the swept area was taken as a function of soil moisture. The exact position of the sewer lines is unknown, the sweep was carried out in parallel plots, separated between them by transverse plots (Figure 4).

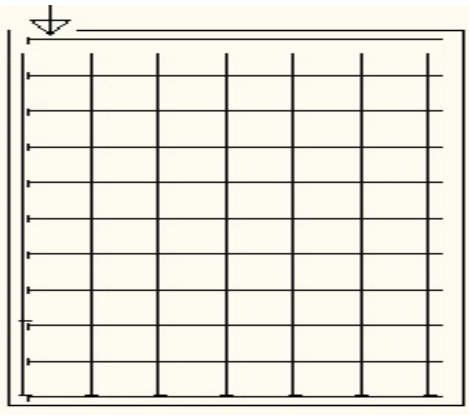

Figure 4: Site GPR Profile Plan

In addition to the GPR profiles, we did a total scan of the area with the radio detector, which allows us to detect buried networks at quite significant depths compared to the GPR up to $5 \mathrm{~m}$ see more if the detector is used in active mode.

\section{RESULTS AND DISCUSSIONS OF INVESTIGATIONS}

Although many GPR profiles have been acquired in the field of study, this paper presents only the most representative results that illustrate the results of the investigations. In order to improve the visualization of the reflections of buried public services, the main steps used in data processing are the following: Zero time correction, a band-pass filter was applied to reduce low and high-frequency noise amplitude and time/depth conversion, based on data obtained by analyzing the geometry of hyperboles associated with the reflections of public services at various depths of the subsoil. All GPR profiles are presented according to depth. 
On the GPR image, from figure 5 we see the presence of reinforcements in the concrete but not on its totality. The presence of steel rebar (rebar) in the concrete structure prevents GPR penetration, where the signal reflects from the top of the structure and returns to the surface. There is also a sudden lateral variation of the physical properties of the subsoil, consisting of a sharp reflection, followed by a GPR reverb pattern. This reflection is due to the presence of an unarmed concrete area that is limited to the entrance of the parking lot, on the rest of the ground the slab is fully armed.

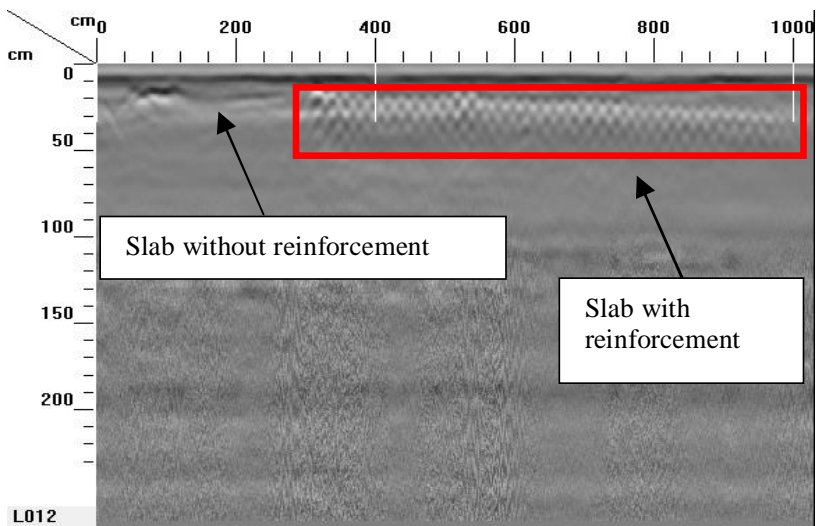

Figure 5: Demonstration of the reinforced concrete slab.

The image obtained from the GPR in Figure 6 shows a subsurface heterogeneity, limited in length and width, as this does not repeat on the other profiles.

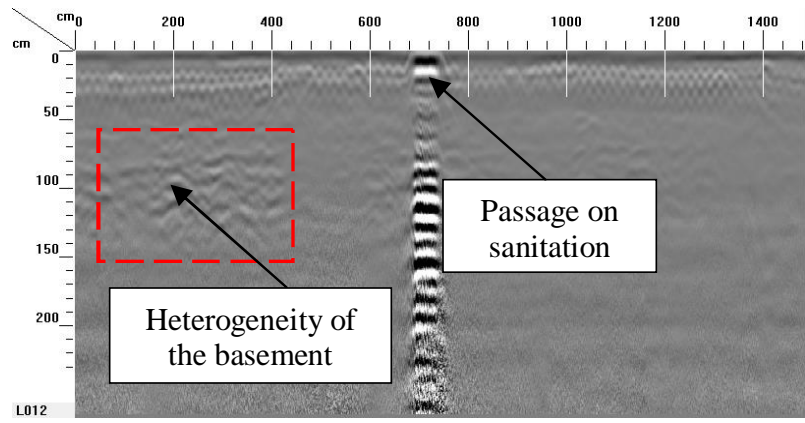

Figure 6: Heterogeneity of the basement

A view of the perpendicular profiles made in this area, confirms the presence of a target in the basement in Figure 7. Because of the high contrast in electrical properties between the ground and the surface like air, the signal is reflected again, and returns to the basement, creating a standard reverb signal that can mask the clear information about the anomaly. This anomaly has been plotted on the drawing in Figure 8. Because of the area, where this anomaly is limited in the basement, we can assume that this could correspond, or the rest of a pipe or the rest of the footings of the old construction that existed on this site.

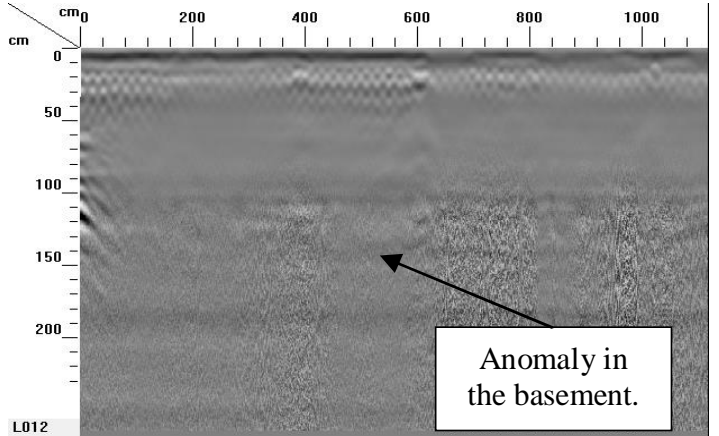

Figure 7: Confirmation of the anomaly

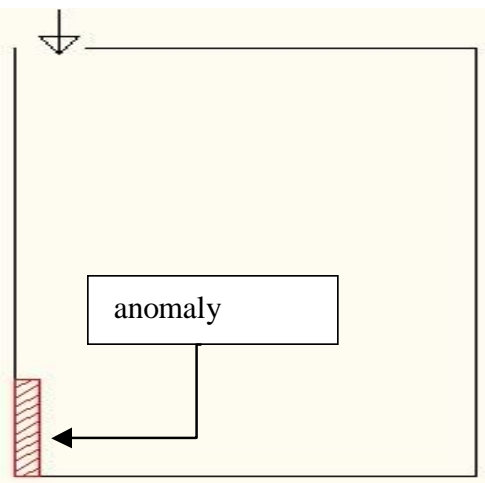

Figure 8: Position of anomaly detected

The various other profiles, such as figure 9, show a pipe repeating on the different parallel profiles. The presence of this pipe in the form of a hyperbolic reflector is clear. The pipeline is confirmed at a depth of $30 \mathrm{~cm}$, which indicates the depth of the top generator.

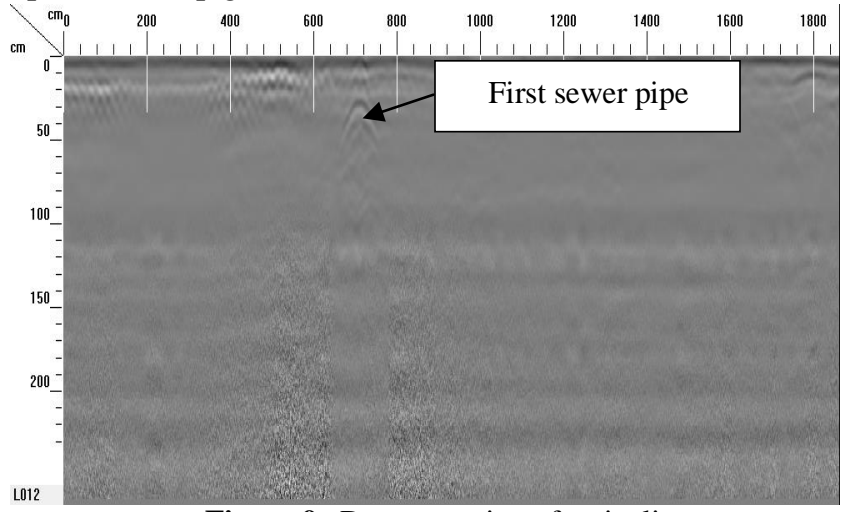

Figure 9: Demonstration of a pipeline

Also the GPR profile shown in Fig 10 shows a pipe detected at the end of the profile, it is not very clear due to the reinforcement density, but it is confirmed on several parallel profiles. This pipe is confirmed at a depth of $50 \mathrm{~cm}$. 


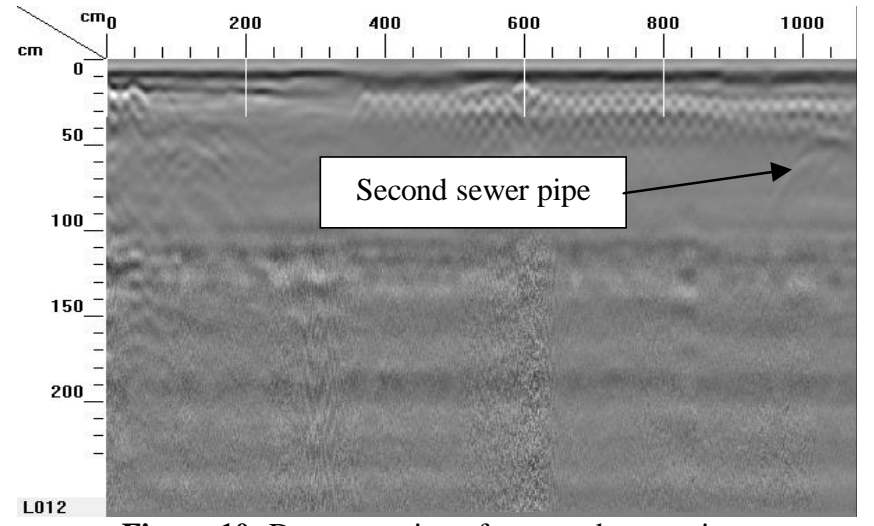

Figure 10: Demonstration of a second sever pipe.

The results of the GPR profile provide an important insight into the shallow subsoil and help locate and estimate utility geometry in the subsoil as shown in figure 11. These results serve as a basis for decision-making and for the practical and rapid implementation of drawing for the collection of buried hydraulic networks and buried cables and the location of anomalies. They also assess the viability of sewer lines and their influence on environmental factors, minimize the risk of hazardous accidents and advance work with greater safety. However, interpretation of these anomalies and confirmation of targets can be done through historical analysis and execution plans as they exist or the creation of boreholes and trenches in well-defined locations.

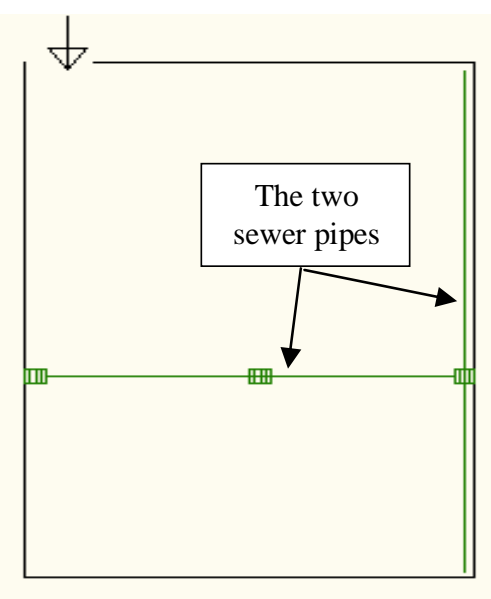

Figure 11: Drawing of underground network

\section{CONCLUSION}

Despite the presence of an armed concrete slab, which could have attenuated the GPR signal, we were able to detect the existing networks and which is limited to a storm water collection network, as indicated in the plan. Hyperbolic reflections of high amplitude characterize targets and pipes. The scanning of the surface by another method (the radio detector), confirms the GPR results.

Apart from the detected pipelines, a surface-limited anomaly was identified and positioned on the plane as part of a study of the GPR profiles for the detection of a pipeline network.
This study shows that the GPR method combined with the radio detector was sufficiently applicable and accurate for practical engineering to precede the detection of anomalies and the precise location of utilities in the basement and the estimation of their depths.

\section{REFERENCES}

1. Metje N, Atkins PR, Brennan MJ, Chapman DN, Lim $\mathrm{HM}$, Machell J, et al. Mapping the underworld state-of-the-art review. Tunn Undergr Space Technol, . 2007.

https://doi.org/10.1016/j.tust.2007.04.002

2. Liu Z, Kleiner Y. State of the art review of inspection technologies for condition assessment of water pipes; Measurement, 2013.

3. Alsharahi, G., A. Faize, M. Louzazni, A. M. M. Mostapha, M. Bayjja, and A. Driouach. Detection of cavities and fragile areas by numerical methods and GPR application. Journal of Applied Geophysics p 164, 225-236, 2019.

https://doi.org/10.1016/j.jappgeo.2019.03.007

4. Alsharahi G., Faize A., Maftei C., Bayjja M., Louzazni M., Driouach A., and Khamlichi A., Analysis and Modeling of GPR Signals to Detect Cavities: Case Studies in Morocco. Journal of Electromagnetic Engineering and Science 19, no. 3, p177-187, 2019. https://doi.org/10.26866/jees.2019.19.3.177

5. Alsharahi G., Mint Mohamed Mostapha A., Modelling and Simulation Resolution of Ground Penetrating Radar Antennas. Journal of electromagnetic engineering and science, 2016.

6. Yogesh P. Deshmukh, and Atul B. Borade. Performance evaluation of the Indian plastic processors supply chain: Implementing lean and green philosophies. International Journal of Emerging Trends in Engineering Research. International Journal, vol. 7, No 5, 2019. https://doi.org/10.30534/ijeter/2019/01752019

7. Singh, Navneet P., Manisha J. Nene, Buried object detection and analysis of GPR images: Using neural network and curve fitting. Annual International Conference on Emerging Research Areas and International Conference on Microelectronics, Communications and Renewable Energy. IEEE, 2013.

8. Pasolli, E., Melgani, F., Donelli, M.. Automatic Analysis of GPR Images: A Pattern-Recognition Approach. Geoscience and Remote Sensing, IEEE Transactions ( 2009).

9. Yang, F., Peng, S.P., Research on the Principle and the Method of Geological Radar Detection. Science press, Bei Jing, 2010.

10. Porsani JL, Ruy YB, Ramos FP, Yamanouth GRB. GPR applied to mapping utilities along the route of the Line 4 (yellow) subway tunnel construction in São Paulo City, Brazil. J Appl Geophys, 2012. https://doi.org/10.1016/j.jappgeo.2012.01.001 
11. Prego, F.J., Solla, M., Puente, I., Arias, P. Efficient GPR data acquisition to detect underground pipes, NDT E Int. 91, 2017.

12. Crocco, L., Prisco, G., Soldovieri, F., Cassidy, N.J. Early-stage leaking pipes GPR monitoring via microwave tomographic inversion. J. Appl. Geophy, 2009.

https://doi.org/10.1016/j.jappgeo.2008.09.006

13. Wang, X.D., He, L., Yang, F., Cao, H. Estimation of soil water content using Ground Penetrating Radar. Chin. J. Geotech. Eng., 2009.

14. Fauchard, C., Dérobert, X., Cariou, J., GPR performances for thickness calibration on road test sites. NDT\&E Int., 2003.

15. Huang, H.W., Du, J., Xie, X.Y. Simulation of GPR detecting of grouting materials behind shield tunnel segments. Chin. J. Geotech. Eng., 2007.

16. Zhang, P., Guo, X., Muhammat, N.,Wang, X.. Research on probing and predicting the diameter of an underground pipeline by GPR during an operation period. Tunn.Undergr. Space Technol, 2016. https://doi.org/10.1016/j.tust.2016.04.005

17. Alani, A.M., and Tosti, F. GPR applications in structural detailing of a major tunnel using different frequency antenna systems, Construction and Building Materials, 2018.

18. Bachiri T., Khamlichi A. \& Bezzazi M., Detection of rebar corrosion in bridge deck by using GPR. In MATEC Web of Conferences, Vol. 191, A.N 00009. NDECS'2017. EDP Sciences, 2018.

19. Bachiri, T., Khamlichi, A., \& Bezzazi, M. Bridge deck condition assessment by using GPR: a review. MATEC Web of Conferences. Vol. 191. A.N 00004. NDECS'2017. EDP Sciences, 2018. https://doi.org/10.1051/matecconf/201819100004

20. Ahmad, M. A., Tvoroshenko, I., Baker, J. H., \& Lyashenko, V. Modeling the Structure of Intellectual Means of Decision-Making Using a System-Oriented NFO Approach. International Journal of Emerging Trends in Engineering Research. International Journal, vol. 7, No 11, 2019. https://doi.org/10.30534/ijeter/2019/107112019

21. Lai WWL, Chang RKW, Sham JFC, Pang K. Perturbation mapping of water leak in buried water pipes via laboratory validation experiments with high-frequency ground penetrating radar (GPR). Tunn Undergr Space Technol, 2016.

22. Glaser DR, Werkema DD, Versteeg RJ, Henderson RD, Rucker DF. Temporal GPR imaging of an ethanol release within a laboratory-scaled sand tank. J Appl Geophys, 2012.

23. W.W.L. Lai, X. Dérobert, P. Annan. A review of Ground Penetrating Radar application in civil engineering: a 30-year journey from locating and testing to imaging and diagnosis. NDT \& E Int., 2017.

24. Birken R, Oristaglio M. Mapping subsurface utilities with mobile electromagnetic geophysical sensor arrays. Sens Technol Civil Infrastruct: Appl Struct HealthMonit, 2014.

https://doi.org/10.1533/9781782422433.2.347
25. Plati C, Dérobert X. Inspection procedures for effective GPR sensing and mapping of underground utilities and voids, with a focus to urban areas. Civil engineering applications of ground penetrating radar. Springer, 2015.

26. Annan AP. Ground penetrating radar applications, principles, procedures. Mississauga, Canada: Sensors and Software, 2004.

27. Cheng NF, Conrad Tang H, Chan CT. Identification and positioning of underground utilities using ground penetrating radar (GPR). Sustain Environ Res, 2013.

28. Jol HM. Ground penetrating radar: theory and applications. Elsevier Science; 2009;

29. Perez-Gracia V, Di Capua D, Gonz_alez-Drigo R, Pujades L. Laboratory characterization of a GPR antenna for high-resolution testing: radiation pattern and vertical resolution. NDT E Int., 2009. https://doi.org/10.1016/j.ndteint.2008.12.007

30. Annan P. Ground penetrating radar: principles, procedures and applications. Mississauga, Canada: Sensors \& Software Inc., 2003.

31. Wang, X.W. Numerical modeling and experiment research of GPR underground pipeline detection. Master Dissertation. Nanjing University of Technology, Nan Jing, 2009. 